\title{
Scattering amplitudes approach to hard thermal loops
}

\author{
Leonardo de la Cruz* \\ Dipartamento di Fisica e Astronomia, Universitá di Bologna and INFN Sezione di Bologna, \\ via Irnerio 46, I-40126 Bologna, Italy \\ and Higgs Centre for Theoretical Physics, School of Physics and Astronomy, \\ The University of Edinburgh, Edinburgh EH9 3FD, Scotland, United Kingdom
}

(Received 28 January 2021; accepted 21 June 2021; published 20 July 2021)

\begin{abstract}
Inspired by recent progress on classical limits of scattering amplitudes, we show that hard thermal loops can be obtained from classical limits of off-shell currents. The classicality of hard thermal loops is made manifest by associating classical wavenumbers to soft particles. We compute the classical limit of these currents in QED, QCD, and gravity. Our proposal does not involve the introduction of ghosts.
\end{abstract}

DOI: $10.1103 /$ PhysRevD.104.014013

\section{INTRODUCTION}

In past decades progress in the field of scattering amplitudes has improved our ability to make predictions in scattering experiments [1]. Recently, techniques developed originally to tackle problems in perturbative quantum field theory are being applied to the study of classical observables and have led to insights about the classical limit, see e.g., [8-24]. We would like to single out two developments in scattering amplitudes. First, a generalization of Feynman's tree theorem [25] (known as loop-tree duality [26-36]) which relates multi-loop amplitudes and phase space integrals and whose integrand is a tree-level amplitudelike object [37]. The loop tree-duality can also be formulated in terms of response functions which can be obtained from the zero temperature Schwinger-Keldysh formalism [27,45]. Second, the novel framework introduced by Kosower-Maybee-O'Connell (KMOC) [11] to study classical observables from amplitudes. The KMOC formalism is based on expectation values of operators between initial states and thus is akin to the zero temperature Schwinger-Keldysh formalism itself.

In this paper we are interested in relating these two ideas to thermal field theory. In their form however we cannot directly apply them since in thermal field theory one is usually interested in currents (rather than scattering amplitudes) from which the thermodynamic properties of the system can be derived. Although the methods we will develop can be applied to QED, QCD and gravity, our

\footnotetext{
*leonardo.delacruz@unibo.it
}

Published by the American Physical Society under the terms of the Creative Commons Attribution 4.0 International license. Further distribution of this work must maintain attribution to the author(s) and the published article's title, journal citation, and DOI. Funded by SCOAP . primary example is a non-Abelian plasma in the high temperature limit.

The limit of high temperature $T$ in perturbative thermal QCD is useful for the description of collective phenomena in plasmas. This limit can be consistently incorporated into an effective theory known as hard thermal loop (HTL) effective theory [46-50]. At the core of HTL effective theory is the resummation of one-loop diagrams with the property of having external soft momenta and internal hard loop momenta. Hard thermal loops (HTLs) are nonlocal currents which are not only gauge invariant but obey simple Ward identities. Assuming a small coupling $g$ the soft momenta are of order $g T$ and the hard momenta of order $T$. HTLs can be computed from the forward scattering of thermal particles [50] which is reminiscent of the loop-tree duality for scattering amplitudes at zero temperature $[26,27]$. On the other hand, HTLs can be reformulated in the language of classical kinetic theory [51,52] and can be obtained from solutions of kinetic equations [53].

The classicality of HTLs and their equivalence with solutions of kinetic equations raises the question of whether we can directly obtain them as a classical limit understood as the limit of $\hbar \rightarrow 0$ of a quantum current. Inspired by the KMOC approach to classical observables, we propose a map between classical limits of off-shell currents at zero temperature and HTL currents. This map is based on the simple observation that to extract classical limits of scattering amplitudes one should distinguish between the momentum $p$ of a particle and its wavenumber $\bar{p}$

$$
p \equiv \hbar \bar{p},
$$

which we interpret as the distinction between soft and hard in HTL currents. As we will see, taking the classical limit will correspond to the high temperature limit. This scaling is the same one required to study the classical dynamics of gravitational waves from quantum field theory. 
Hence we will mostly use the KMOC approach but other strategies to obtain the classical limit may be used as well. e.g., [12,20,54].

\section{FROM KINETIC THEORY TO CLASSICAL LIMITS OF OFF-SHELL CURRENTS}

The dynamics of non-Abelian plasmas is described by QCD at finite temperature at distances $\hbar / T \lesssim d \lesssim \hbar /(g T)$. For the rest of the paper we will use units in which $k_{B}=c=1$, but keep $\hbar \neq 1$ since we are interested in the study of the classical limit. The leading-order thermal effects can be studied employing relativistic kinetic theory, which is applicable at scales where the average distance between particles is of order $\hbar / T$ and such that the plasma is characterized by the Debye length $\hbar /(g T)$. In the microscopic approach to kinetic theory [55], we are interested in an ensemble of pointlike particles characterized by a phase-space distribution $f \equiv f(x, p, c)$, where the color degrees of freedom $c$ of the plasma are treated as continuous classical variables. The phase space variables obey Wong equations [59]

$$
\begin{gathered}
\frac{\mathrm{d} k^{\mu}}{\mathrm{d} \tau}=g c^{a}(\tau) F^{a \mu \nu}(x(\tau)) v_{\nu}(\tau), \\
\frac{\mathrm{d} c^{a}}{\mathrm{~d} \tau}=g f^{a b c} v^{\mu}(\tau) A_{\mu}^{b}(x(\tau)) c^{c}(\tau), \\
D^{\mu} F_{\mu \nu}^{a}(x)=J_{\nu}^{a}(x)=g \int \mathrm{d} \Phi(k) \mathrm{d} c k_{\nu} c^{a} f(x, k, c),
\end{gathered}
$$

where the Lorentz invariant phase space measure is defined by $\mathrm{d} \Phi(k) \equiv \frac{\mathrm{d}^{4} k}{(2 \pi)^{4}} \Theta\left(k_{0}\right) 2 \pi \delta\left(k^{2}-m^{2}\right)$. Specializing to the case of $S U(3)$ the color measure is defined by $\mathrm{d} c \equiv \mathrm{d}^{8} c c_{R} \delta\left(c^{a} c^{a}-q_{2}\right) \delta\left(d_{a b c} c^{a} c^{b} c^{c}-q_{3}\right)$, where the color part ensures the conservation of the Casimir invariants $q_{i}$, and $c_{R}$ is a normalization constant defined such that $\int \mathrm{d} c=1$ [60]. In the collisionless case the Vlasov-type equation for the distribution function $f(x, k, c)$ is given by

$$
\frac{\mathrm{d} f}{\mathrm{~d} \tau}=k^{\mu}\left(\frac{\partial}{\partial x^{\mu}}-g f^{a b c} A_{\mu}^{b} c^{c} \frac{\partial}{\partial c^{a}}-g c^{a} F_{\mu \nu}^{a} \frac{\partial}{\partial k_{\nu}}\right) f=0 .
$$

It is well known that both Wong and Vlasov equations can be derived from suitable classical limits [61]. The Vlasov equations arises from the classical limit of the corresponding equation for the Wigner operator [62-64].

In this paper however, we are interested in the classical limits of currents leading to solutions of the kinetic equations rather than a derivation of kinetic equations themselves. Equation (5) can be solved iteratively by expanding $f(x, k, c)$ around the equilibrium state. In equilibrium the distribution function only depends on the energy $k_{0}$ of the system and we can expand it in powers of the coupling $g$

$$
f(x, k, c)=f^{(0)}\left(k_{0}\right)+\Delta^{(1)} f(x, k, c)+\ldots,
$$

where $\Delta^{(i)} f(x, k, c)$ is of order $\mathcal{O}\left(g^{i}\right)$. We can then use the equilibrium distribution function to solve for $\Delta^{(1)} f(x, k, c)$ and reinsert it to find $\Delta^{(2)} f(x, k, c)$ and so on. From Eq. (4) we can write the total current as [65]

$$
J_{a}^{\mu}(x)=\Delta^{(1)} J_{a}^{\mu}(x)+\Delta^{(2)} J_{a}^{\mu}(x)+\ldots
$$

The currents can be expressed in the form

$$
J_{\mu}^{a}(x)=\Pi_{\mu \nu}^{a b} A_{b}^{\nu}+\frac{1}{2} \Pi_{\mu \nu \rho}^{a b c} A_{b}^{\nu} A_{c}^{\rho}+\ldots,
$$

where $\Pi_{\mu \nu \cdots}^{a b \cdots}$ are thermal currents [66], which can be matched against those obtained from the high temperature limit of thermal QCD (See e.g., Ref. [67]). One can thus conclude that HTL are classical $[53,68]$. Notice that despite our choice of units we will keep our classical quantities independent of $\hbar$ as they should. The setup leading to Eq. (7) is reminiscent of the one to obtain perturbative solutions from classical equations of motion [54,69-75] which can be matched to classical limits of scattering amplitudes. Within the KMOC formalism this is done by defining certain observables which are well defined both classically and quantum mechanically $[11,16,76]$. Besides the distinction between the momentum $p$ of a particle and its wavenumber $\bar{p}$ the introduction of appropriate coherent states is required to take the classical limit. Since we are interested in off-shell currents rather than observables, we cannot directly apply the KMOC formalism but as we will see many of its characteristics remain.

The classical limit of amplitudes in the KMOC formalism is slightly different in QED and QCD due to the presence of color in the latter. In QCD the dimensionless coupling $g$ scales as $\bar{g} \sqrt{\hbar}$. In QED the dimensionless coupling $e$ scales as $\bar{e} / \sqrt{\hbar}$ and similarly for gravity [77]. The scaling in QCD is complemented by scaling of the color factors, so ultimately we can use the same procedure in Ref. [11] to obtain the classical limit. Adopting the conventions of Ref. [76] we have

$$
\left[\mathbb{C}^{a}, \mathbb{C}^{b}\right]=\mathrm{i} \hbar f^{a b c} \mathbb{C}^{c},
$$

emphasizing that $\mathbb{C}$ corresponds to an operator and $\left\langle p_{i}\left|\mathbb{C}^{a}\right| p^{j}\right\rangle \equiv\left(C^{a}\right)_{i}^{j}=\hbar\left(T^{a}\right)_{i}{ }_{i}$. The corresponding classical color charge $c^{a} \equiv\left\langle\psi\left|\mathbb{C}^{a}\right| \psi\right\rangle$ is obtained from appropriate coherent states $|\psi\rangle$. In the spinless case gravity does not bring any new ingredients other than complexity so the KMOC algorithm follows the QED one.

The interplay between soft and hard momenta is very generally linked to the classical limit, usually through the Eikonal approximation, and thus it is tempting to relate it the HTL approximation. However, operationally in the HTL approximation one integrates over hard momenta 
running in the loop $k \sim T$ and considers external soft momenta $p \sim g T$. The results are then expanded in powers of $|p| /|k|$, see Ref. [67] for a summary of the rules to compute HTLs. By associating classical wavenumbers to the external soft momenta such that they scale with $\hbar$, an expansion in powers of $\hbar$ will correspond to an expansion in high temperature. This formal equivalence can also be observed at the level of the dimensional reduced effective action [78]. Physically this has the interpretation of matching the high temperature regime to the classical regime.

In momentum space the coefficients in Eq. (8) can be written as follows $[50,80]$ :

$$
\Pi_{\mu_{1} \mu_{2} \cdots \mu_{n}}^{a_{1} a_{2} \ldots a_{n}}(p)=\int \mathrm{d} \Phi(k) N\left(k_{0}\right) \mathcal{H}_{\mu_{1} \mu_{2} \cdots \mu_{n}}^{a_{1} a_{2} \ldots a_{n}}(k, p)
$$

where $N\left(k_{0}\right)$ is some distribution function and $\mathcal{H}_{n} \equiv$ $\mathcal{H}_{\mu_{1} \mu_{2} \cdots \mu_{n}}^{a_{1} a_{2} \ldots a_{n}}(k, p)$ is the integrand of the HTL current. If we are interested in fermions we will define the distribution function with a minus sign due to the presence of a fermion loop. We have used the shorthand notation $p$ to express the dependence on the full set of external momenta $p_{1}, \ldots, p_{n}$. A generating functional has been given in Refs. [49,81].

In thermal perturbation theory the integrands in Eq. (10) can be constructed by considering permutations of comb diagrams (See Fig. 1). This approach can be applied systematically to a variety of theories including gravity [45,88-95]. For on-shell amplitudes at zero temperature Eq. (10) is the usual forward limit $[26,27,45]$ and hence a current at zero temperature is a natural candidate to relate the classical limit and the high temperature limit. Therefore, we consider the current represented in Fig. 2 which can be computed using the Feynman rules of the theory under consideration. These currents have the property of having $n$ particles off shell while the momentum of the massive particle running in the loop is on shell, that is $k^{2}=m^{2}$.

The forward limit is in general singular; therefore we must consider a regularization scheme. We define $\mathcal{F}$ as the set of all Feynman graphs and $\mathcal{S}$ as the set of tadpole graphs-i.e., those graphs containing a zero-momentum internal edge (see Fig. 3). Suppressing color and Lorentz indices, we consider the following regularized current

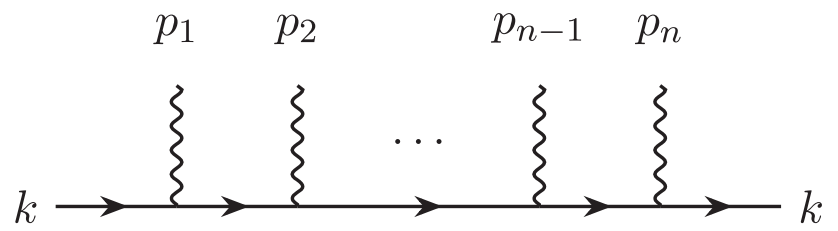

FIG. 1. Typical comb diagrams required for the calculation of currents in the high temperature limit. The wavy lines represent gauge bosons. The solid lines represent massive particles such as scalars or quarks, or massless particles such as gauge bosons or ghosts.

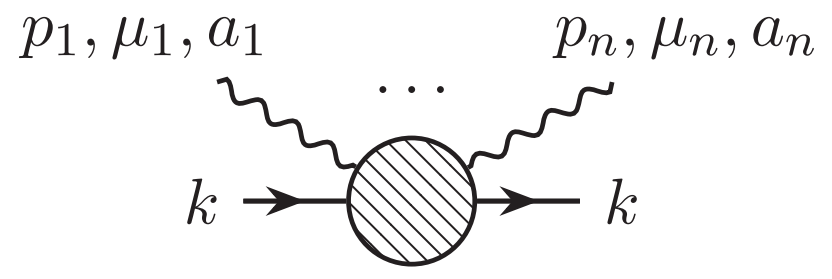

FIG. 2. Off-shell current. The blob represents a sum over treelevel Feynman diagrams. Off-shell gauge bosons can be photons, gluons or gravitons.

$$
\mathcal{A}_{n}\left(k, p_{1}, \ldots, p_{n}, k\right) \equiv \sum_{G \in \mathcal{F} \backslash \mathcal{S}} f(G),
$$

where $f(G)$ is a rational expression of the form $N(G) / D(G)$. The regularized current is simply obtained by removing tadpole graphs [96]. In the following we assume that the current is regulated. The classical limit of this current will be defined by

$\overline{\mathcal{A}}_{n}\left(k, \bar{p}_{1}, \ldots, \bar{p}_{n}\right) \equiv \widetilde{\operatorname{Tr}}\left(\lim _{\hbar \rightarrow 0} \mathcal{A}_{n}\left(k, \hbar \bar{p}_{1}, \ldots, \hbar \bar{p}_{n}, k\right)\right)$,

which is obtained by performing a Laurent expansion in powers of $\hbar$ after rescaling the momenta of the soft particles and couplings following the algorithms in Refs. [11,16,76]. The operator $\widetilde{T r}$ depends on whether the theory is colored or not. We define it by

$$
\widetilde{\operatorname{Tr}}(\bullet) \equiv \begin{cases}\hbar^{n-2} \operatorname{Tr}(\bullet) & \text { QCD, } \\ \operatorname{Id}(\bullet) & \text { QED and gravity, }\end{cases}
$$

where Id is the identity operator and the $\hbar^{n-2}$ is required on dimensional grounds. We adopt the convention that our classical results will depend on the dimensionless coupling $g=\bar{g} \sqrt{\hbar}$ and $e=\bar{e} / \sqrt{\hbar}$, and that the external momenta is associated to wavenumbers. If desired one may restore the dependence on momenta by dimensional analysis. In the case of QCD, this requires an overall inverse power of $\hbar$ in Eq. (13). The currents just defined are in general gauge dependent. Similar currents occur for example in the Berends-Giele recursion [97].

We propose mapping the integrand in (10) in momentum space to the classical limit of the $n$-point forward current

$$
\overline{\mathcal{A}}_{n}\left(k, \bar{p}_{1}, \ldots, \bar{p}_{n}\right) \leftrightarrow \mathcal{H}_{n}\left(k, p_{1}, \ldots, p_{n}\right),
$$

and similarly for QED and gravity.

Using the imaginary-time formalism, where time is traded with the temperature and the structure of the propagator is similar to the zero temperature case, we can relate zerotemperature amplitudes and finite-temperature amplitudes by analytic continuation (see e.g., Ref. [98]). The currents we are proposing are amplitudelike objects and their computation is not based on sums over permutations of 
comb diagrams (see Fig. 1) as the usual forward scattering approach to thermal field theory [50]. Let us also remark that although we are considering the case where only massive particles appear in the loop, this case can also be utilized to study the case in which hard gauge particles run in the loop since the tensor structures are the same [99]. Thus, it is enough to discuss the case with matter loops. The pure gauge calculation with ghosts included appears e.g., in Ref. [45]. It is in this sense that ghosts will not be required. From an amplitudes point of view this is a simple consequence of our calculation being a tree-level one.

In order to model the non-Abelian plasma we use the Lagrangian

$$
\mathcal{L}=\left[\left(D_{\mu} \varphi^{\dagger}\right) D^{\mu} \varphi-\frac{m^{2}}{\hbar^{2}} \varphi^{\dagger} \varphi\right]-\frac{1}{4} F_{\mu \nu}^{a} F^{a \mu \nu},
$$

where $D_{\mu}=\partial_{\mu}+i g A_{\mu}^{a} T^{a}$, and the field strength tensor $F_{\mu \nu}^{a}=\partial_{\mu} A_{\nu}^{a}-\partial_{\nu} A_{\mu}^{a}-g f^{a b c} A_{\mu}^{b} A_{\nu}^{c}$. For QED we employ the Abelian version of this Lagrangian. Let us remark that Eq. (5) and its Abelian version describe a plasma for spinless particles and therefore this Lagrangian is the appropriate one to consider in the classical limit [100]. We use the Feynman gauge for computations and adopt the following normalization of the generators of the Lie algebra

$$
\operatorname{Tr}\left(C^{a} C^{b}\right)=\frac{\hbar^{2}}{2} \delta^{a b}
$$

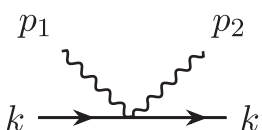

(a)

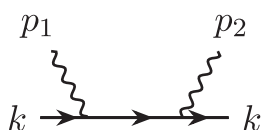

(b)

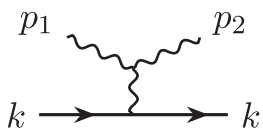

(d)

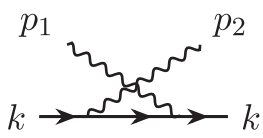

(c)
FIG. 3. Diagrams related to the two-point function in QED, QCD and gravity: Diagrams (a)-(c) contribute to the regularized forward limit. Diagram (d) leads to a one-loop diagram with zero momentum, i.e., a tadpole.

\section{QED}

As our first example we will consider a QED plasma where the equilibrium distribution is given by the FermiDirac distribution $N\left(k_{0}\right) \equiv-1 /\left(\exp \left(k_{0} / T\right)+1\right)$, and where the sign is included to indicate that we are interested in matching the high temperature limit of $n$-point functions in QED with a fermion running in the loop. Recall that in our approach, however, the classical limit is taken from scalar QED since we are solving the spinless Vlasov equation. The regulated current is simply $\mathcal{A}_{n}\left(k, \hbar \bar{p}_{1}, \ldots, \hbar \bar{p}_{n}, k\right)$, where all photons are outgoing. Let us consider the simple case $n=2$ whose contributing diagrams are shown in Fig. 3. Using the rescaled momenta a simple calculation leads to

$$
\mathcal{A}^{\mu \nu}\left(k, \hbar \bar{p}_{1}, \hbar \bar{p}_{2}, k\right)=2 e^{2}\left(\eta^{\mu \nu}+\frac{k^{\nu}\left(4 \bar{p}_{1}{ }^{2} k^{\mu}-4 k \cdot \bar{p}_{1} \bar{p}_{1}{ }^{\mu}\right)-4 k^{\mu} k \cdot \bar{p}_{1} \bar{p}_{1}{ }^{\nu}+\hbar^{2} \bar{p}_{1}{ }^{2} \bar{p}_{1}{ }^{\mu} \bar{p}_{1}{ }^{\nu}}{4\left(k \cdot \bar{p}_{1}\right)^{2}-\hbar^{2}\left(\bar{p}_{1}{ }^{2}\right)^{2}}\right),
$$

where we have used $p_{2}=-p_{1}$. Upon performing a Laurent expansion in powers of $\hbar$ we obtain

$\overline{\mathcal{A}}^{\mu \nu}(k, \bar{p})=2 e^{2}\left(\eta^{\mu \nu}+\frac{\bar{p}_{1}{ }^{2} k^{\mu} k^{\nu}}{\left(k \cdot \bar{p}_{1}\right)^{2}}-\frac{k^{\nu} \bar{p}_{1}{ }^{\mu}+k^{\mu} \bar{p}_{1}{ }^{\nu}}{k \cdot \bar{p}_{1}}\right)$,

which recovers the $\mathcal{O}\left(T^{2}\right)$ HTL two-photon integrand.

Notice that the propagators considered for our currents do not have an i $\epsilon$ term. In order to recover the retarded temperature dependent currents we consider the analytic continuation $\bar{p}_{n}^{0} \rightarrow \bar{p}_{n}^{0}+\mathrm{i} \epsilon$ and $\bar{p}_{i}^{0} \rightarrow \bar{p}_{i}^{0}-\mathrm{i} \epsilon$, for $i=1, \ldots, n-1$, where we assume that the vertex corresponding to $\bar{p}_{n}$ corresponds to the one with the largest time [91]. Finally, we can perform the radial integration by parametrizing $k^{\mu}=|\mathbf{k}| K^{\mu} \quad$ with $K^{\mu}=$ $\left(\sqrt{1+m^{2} /|\mathbf{k}|^{2}}, \mathbf{k} /|\mathbf{k}|\right)[45,88,95]$ (see Appendix A).

This simple example summarizes our approach in general: first consider the $n$-point regulated current (12), then compute the current using Feynman graphs or Berends-Giele recursions, etc., and calculate the classical limit with the replacement $\hbar \bar{p}_{i}$ for the gauge bosons. Physical propagators are obtained by analytic continuation.

In Ref. [50] "superleading" terms in the temperature expansion appear, which cancel after all permutations of graphs are considered. In our approach we are not considering permutations (e.g., in the $n=2$ case we are only summing 3a-3c in Fig. 3) so it is interesting to ask if "superleading"terms appear here too. In our approach these terms have the form $k^{\mu_{1}} \cdots k^{\mu_{n}} /\left(\hbar^{n-1}\left(k \cdot \bar{p}_{1}\right) \cdots\left(k \cdot \bar{p}_{n-1}\right)\right)$ and hence they correspond to (if any) singular terms in the classical limit. In our general expression in Eq. (12) these terms cancel (QED) or vanish (QCD). The HTL currents truly arise as classical limits of the currents $\mathcal{A}^{\mu \nu}\left(k, \hbar \bar{p}_{1}, \hbar \bar{p}_{2}, k\right)$. For a neutral QED plasma it is well known that higher point functions are either vanishing or subleading (see e.g., Ref. [102]). We can however consider a non-neutral plasma which would still be described by the Vlasov equation and thus study generalizations of HTL for higher point functions in QED [95]. After taking the classical limit and keeping terms of $\mathcal{O}\left(\hbar^{0}\right)$ it is straightforward to compute higher-order corrections in the temperature. 
We have checked up to $n=4$ that our methods reproduce those obtained from the thermal field theory approach [95]. (A three-point example is given in Appendix B.)

\section{QCD AND GRAVITY}

The QED case illustrates the procedure of taking the classical limit for the kinematics. The new ingredient in
QCD is color. The lowest-order contribution requires the calculation of a diagram with a three-gluon vertex as shown in Fig. 3, which leads to a tadpole diagram and therefore removed [103]. Notice that the first diagram in Fig. 3 does not lead to a tadpole since the internal edge with zero momentum is not present. Computing the current and taking the classical limit leads to

$$
\begin{aligned}
\mathcal{A}_{a_{1} a_{2}}^{\mu_{1} \mu_{2}}(k, \hbar \bar{p})= & \frac{\bar{g}^{2}}{\hbar}\left[C^{a_{1}} \cdot C^{a_{2}}\left(\eta^{\mu_{1} \mu_{2}}-\frac{2 k^{\mu_{1}} k^{\mu_{2}}}{\hbar k \cdot \bar{p}_{1}}+\frac{\bar{p}_{1}{ }^{2} k^{\mu_{1}} k^{\mu_{2}}}{\left(k \cdot \bar{p}_{1}\right)^{2}}-\frac{k^{\nu} \bar{p}_{1}{ }^{\mu}}{k \cdot \bar{p}_{1}}-\frac{k^{\mu_{1}} \bar{p}_{1}^{\mu_{2}}}{k \cdot \bar{p}_{1}}\right)\right. \\
& \left.+C^{a_{2}} \cdot C^{a_{1}}\left(\eta^{\mu_{1} \mu_{2}}+\frac{2 k^{\mu_{1}} k^{\mu_{2}}}{\hbar k \cdot \bar{p}_{1}}+\frac{\bar{p}_{1}{ }^{2} k^{\mu_{1}} k^{\mu_{2}}}{\left(k \cdot \bar{p}_{1}\right)^{2}}-\frac{k^{\mu_{2}} \bar{p}_{1}{ }^{\mu_{1}}}{k \cdot \bar{p}_{1}}-\frac{k^{\mu_{1}} \bar{p}_{1}^{\mu_{2}}}{k \cdot \bar{p}_{1}}\right)+\mathcal{O}(\hbar)\right],
\end{aligned}
$$

where $C^{a} \cdot C^{b} \equiv\left(C^{a}\right)^{i}{ }_{j}\left(C^{b}\right)^{j}{ }_{k}$. At first sight this expression contains singular terms of the form $k^{\mu_{1}} k^{\mu_{2}} /\left(\hbar k \cdot \bar{p}_{1}\right)$, which we mentioned previously. However using Lie algebra (9) these produce classical terms with a vanishing trace. Therefore after tracing we obtain the well-known result for QCD

$$
\overline{\mathcal{A}}_{a_{1} a_{2}}^{\mu_{1} \mu_{2}}(k, \bar{p})=\delta^{a_{1} a_{2}} \Pi^{\mu_{1} \mu_{2}}\left(\bar{p}_{1}\right) \equiv \delta^{a_{1} a_{2}} g^{2}\left(\frac{\bar{p}_{1}^{2} k^{\mu_{1}} k^{\mu_{2}}}{\left(k \cdot \bar{p}_{1}\right)^{2}}-\frac{k^{\mu_{2}} \bar{p}_{1}{ }_{1}^{\mu_{1}}}{k \cdot \bar{p}_{1}}-\frac{k^{\mu_{1}} \bar{p}_{1}^{\mu_{2}}}{k \cdot \bar{p}_{1}}+\eta^{\mu_{1} \mu_{2}}\right) .
$$

Notice that the final result is expressed in terms of the dimensionless coupling $\bar{g} \sqrt{\hbar}$. We now consider the three-point function. We decompose the current in a basis of color factors as follows:

$$
\mathcal{A}_{a_{1} a_{2} a_{3}}^{\mu_{1} \mu_{2} \mu_{3}}(k, \hbar \bar{p}, k)=\sum_{\sigma \in S_{3}} C^{a_{\sigma_{1}}} \cdot C^{a_{\sigma_{2}}} \cdot C^{a_{\sigma_{3}} J^{\mu_{1} \mu_{2} \mu_{3}}}\left(\sigma_{1}, \sigma_{2}, \sigma_{3}\right)
$$

The kinematic coefficients $J^{\mu_{1} \mu_{2} \mu_{3}}\left(\sigma_{1}, \sigma_{2}, \sigma_{3}\right)$ are straightforward to compute-but lengthy and not presented here. The leading contribution in the classical limit is of $\operatorname{order} \mathcal{O}(1 / \hbar)$, i.e., singular at first sight. However upon tracing we can bring the result into the form

$$
\overline{\mathcal{A}}_{a_{1} a_{2} a_{3}}^{\mu_{1} \mu_{2} \mu_{3}}(k, \bar{p})=2 \frac{\bar{g}^{3} \hbar}{\hbar^{3 / 2}}\left[\operatorname{Tr}\left(C^{a_{1}} \cdot C^{a_{3}} \cdot C^{a_{2}}\right)-\operatorname{Tr}\left(C^{a_{1}} \cdot C^{a_{2}} \cdot C^{a_{3}}\right)\right] \frac{A_{\mathrm{QED}}^{\mu_{1} \mu_{2} \mu_{3}}}{\hbar}
$$

where

$$
A_{\mathrm{QED}}^{\mu_{1} \mu_{2} \mu_{3}}=\sum_{\sigma \in \mathrm{Cyclic}}\left[\frac{2 k^{\mu_{\sigma_{1}}} k^{\mu_{\sigma_{2}}}}{k \cdot \bar{p}_{\sigma_{3}}}\left(\frac{\bar{p}_{\sigma_{1}}^{\mu_{\sigma_{3}}}}{k \cdot \bar{p}_{\sigma_{1}}}-\frac{\bar{p}_{\sigma_{2}}^{\mu_{\sigma_{3}}}}{k \cdot \bar{p}_{\sigma_{2}}}\right)+k^{\mu_{\sigma_{1}}} k^{\mu_{\sigma_{2}}} k^{\mu_{\sigma_{3}}}\left(\frac{\bar{p}_{\sigma_{1}}^{2}}{\left(k \cdot \bar{p}_{\sigma_{1}}\right)^{2}}\left(\frac{1}{k \cdot \bar{p}_{\sigma_{2}}}-\frac{1}{k \cdot \bar{p}_{\sigma_{3}}}\right)\right)\right],
$$

where Cyclic is the set of cyclic permutations of $\{1,2,3\}$. Notice that in the commutative case Eq. (21) is simple telling us that singular terms vanish in the classical limit since the operator $\widetilde{\mathrm{Tr}}$ is replaced by the identity operator. Using Eqs. (9) and (16) we find

$$
\overline{\mathcal{A}}_{a_{1} a_{2} a_{3}}^{\mu_{1} \mu_{2} \mu_{3}}(k, \bar{p})=\mathrm{i} g^{3} f^{a_{1} a_{2} a_{3}} A_{\mathrm{QED}}^{\mu_{1} \mu_{2} \mu_{3}},
$$

where the current satisfies the identity

$$
\bar{p}_{3 \mu_{3}} \overline{\mathcal{A}}_{a_{1} a_{2} a_{3}}^{\mu_{1} \mu_{2} \mu_{3}}(k, \bar{p})=\mathrm{i} g f^{a_{1} a_{2} a_{3}}\left[\Pi^{\mu_{1} \mu_{2}}\left(p_{1}\right)-\Pi^{\mu_{1} \mu_{2}}\left(p_{2}\right)\right] .
$$

Remarkably, we find that the kinematic structure of the current is encoded in the commutative QED part in a similar way as the classical color and momentum impulse observables [76]. Although we can continue computing the next contributions in a similar fashion, it is well known that we can reconstruct higher-point functions using Ward identities and the above relation $[47,48]$. The expression (22) agrees with previously computed expressions in Refs. [93,104].

There is no new ingredient in gravity regarding the classical limit and therefore we can apply our formalism for this case too. HTLs for gravity (reviewed in Ref. [105]) are relevant for the physics of the early Universe. They have 
been investigated in Refs. $[89,106]$. We expand in powers of the graviton coupling $\kappa$ with $\kappa^{2}=32 \pi G$ and set $g_{\mu_{1} \mu_{2}}=\eta_{\mu_{1} \mu_{2}}+\kappa h_{\mu_{1} \mu_{2}}$. The two-point graviton function can be computed from the diagrams in Fig. 3 with the gluon replaced by a graviton, where as in QCD we regulate the current by removing contributions from the threegraviton vertex. Using the conventions in Ref. [107] the calculation is straightforward and leads to

$$
\begin{aligned}
\overline{\mathcal{A}}^{\alpha \beta ; \gamma \delta}(k, \bar{p})= & \frac{1}{2} \kappa^{2}\left[\frac{\bar{p}_{1}{ }^{2} k^{\alpha} k^{\beta} k^{\gamma} k^{\delta}}{\left(k \cdot \bar{p}_{1}\right)^{2}}-\frac{k^{\beta} k^{\gamma} k^{\delta} \bar{p}_{1}{ }^{\alpha}+k^{\alpha} k^{\gamma} k^{\delta} \bar{p}_{1}{ }^{\beta}+k^{\alpha} k^{\beta} k^{\delta} \bar{p}_{1}{ }^{\gamma}-k^{\alpha} k^{\beta} k^{\gamma} \bar{p}_{1}{ }^{\delta}}{k \cdot \bar{p}_{1}}\right. \\
& \left.+\eta^{\beta \gamma} k^{\alpha} k^{\delta}+\eta^{\beta \delta} k^{\alpha} k^{\gamma}+\eta^{\alpha \gamma} k^{\beta} k^{\delta}+\eta^{\alpha \delta} k^{\beta} k^{\gamma}\right],
\end{aligned}
$$

which agrees with the result in Ref. [89]. In general the two-point graviton function depends on the representation of the graviton field but one can redefine it in terms of the above expression to obtain a two-point function independent of the graviton parametrization [89].

\section{DISCUSSION}

In this paper we have shown that HTLs arise from classical limits of off-shell currents. The classical nature of hard-thermal loop amplitudes was made manifest by relating the momenta of the soft particles to wavenumbers. The classical limit is then obtained following the KMOC algorithm. In this way, the high temperature limit is formally equivalent to an expansion in powers of $\hbar$ thus allowing a map between HTL amplitudes and classical limits of offshell currents. The off-shell currents encode the information of permutation of comb diagrams and can be easily computed from Feynman diagrams or Berends-Giele recursions. Since our off-shell currents in the classical limit are gauge invariant and satisfy Ward identities, their "on-shell" properties would be interesting to study, in particular the Britto-Cachazo-Feng-Witten [108] recursion, the colorkinematics duality and the double copy [109-111]. For general off-shell currents these properties are generally more difficult to make manifest than for amplitudes [112-114]. At the classical level the double copy is much more flexible but the idea of a formal replacement between color and kinematics is preserved [115]. This classical double copy is appropriate to relate gravity and QCD in the high temperature limit.

On the more phenomenological side the inclusion of spin and collisions [116] would be relevant for a more complete description of a non-Abelian plasma from kinetic theory. Vlasov equations with spin can be obtained from first principles using Wigner functions and thus it would be interesting to describe currents with spin. (See e.g., $[120,121]$ for a recent application of Wigner functions in the case with collisions.) The KMOC formalism has been applied to the spin cases in Ref. [16]. In principle, the spin case would require considering classical limits of spinor wavefunctions. Collision functions in kinetic theory can be obtained from Wigner functions, which can be computed using scattering amplitudes [122]. Perhaps one can study the inclusion of collisions considering classical limits of scattering amplitudes within Wigner functions.

\section{ACKNOWLEDGMENTS}

We thank Ben Maybee, Donal O'Connell, and Alasdair Ross for many discussions on the KMOC formalism. We thank Ben Maybee and Donal O'Connell for useful comments on the manuscript. We thank Fernando T. Brandt for useful correspondence and Luis A. Hernandez for informative discussions. This work was partially supported by the STFC Grant No. ST/P0000630/1. The author acknowledges financial support from the Open Physics Hub at the Physics and Astronomy Department in Bologna. Our figures were produced with the help of TikZ-Feynman [123]. Some of the calculations in this paper were done with Feyncalc [124-126].

\section{APPENDIX A: TEMPERATURE DEPENDENCE}

The temperature can be recovered following the procedure in Ref. [95], where the reader can check details. For simplicity, let us consider the two-point QED example and consider Eq. (10). Using our map, we have

$$
\begin{aligned}
\Pi^{\mu \nu}(\bar{p})= & \int \frac{\mathrm{d}^{4} k}{(2 \pi)^{4}} \theta\left(k_{0}\right) 2 \pi \delta\left(k^{2}-m^{2}\right) \\
& \times N\left(k_{0}\right) \overline{\mathcal{A}}_{2}^{\mu \nu}(k, \bar{p}),
\end{aligned}
$$

which leads to

$\Pi^{\mu \nu}(\bar{p})=\left.\frac{1}{(2 \pi)^{3}} \int \frac{\mathrm{d}^{3} \mathbf{k} N\left(\sqrt{\mathbf{k}^{2}+m^{2}}\right)}{2 \sqrt{\mathbf{k}^{2}+m^{2}}} \overline{\mathcal{A}}_{2}^{\mu \nu}(k, \bar{p})\right|_{k_{0}=+\sqrt{\mathbf{k}^{2}+m^{2}}}$.

Let us define $k_{0}=|\mathbf{k}| K_{0}$ with $K_{0}>0$ and use spherical coordinates 


$$
\Pi^{\mu \nu}(\bar{p})=\left.\frac{1}{(2 \pi)^{3}} \int \frac{\mathrm{d}|\mathbf{k}||\mathbf{k}|^{2} N\left(|\mathbf{k}| K_{0}\right)}{2|\mathbf{k}| K_{0}} \int \mathrm{d} \Omega \overline{\mathcal{A}}_{2}^{\mu \nu}(k, \bar{p})\right|_{k_{0}=|\mathbf{k}| K_{0}} .
$$

We can simplify the above expression by introducing the unit vector vector $\hat{\mathbf{K}}=\mathbf{k} /|\mathbf{k}|$ and defining the four vector

$$
K^{\mu}=\left(k_{0} /|\mathbf{k}|, \mathbf{k} /|\mathbf{k}|\right),
$$

such that $k^{\mu}=|\mathbf{k}| K^{\mu}$. Since $\overline{\mathcal{A}}_{2}^{\mu \nu}(k, \bar{p})$ is a homogeneous function of degree zero in $k$ we can then write

$$
\Pi^{\mu \nu}(\bar{p})=\frac{1}{(2 \pi)^{3}} \int_{0}^{\infty} \frac{\mathrm{d}|\mathbf{k}||\mathbf{k}|^{2} N\left(|\mathbf{k}| K_{0}\right)}{2|\mathbf{k}| K_{0}} \int \mathrm{d} \Omega \overline{\mathcal{A}}_{2}^{\mu \nu}(K, \bar{p}) .
$$

Using $K_{0}=\sqrt{1+m^{2} /|k|^{2}}$ and the change of variables $x=|\mathbf{k}| / T$ leads to

$$
\Pi^{\mu \nu}(\bar{p})=-\frac{1}{(2 \pi)^{3}} I(T, m) \int \mathrm{d} \Omega \overline{\mathcal{A}}_{2}^{\mu \nu}(K, \bar{p}),
$$

where we have included a minus sign to indicate that we are interested in the classical limit where a fermion is running in the loop. The temperature dependence is then obtained from

$$
I(T, m) \equiv T^{2} \int_{0}^{\infty} \mathrm{d} x \frac{x^{2}}{2 \sqrt{x^{2}+\frac{m^{2}}{T^{2}}} \exp \left(\sqrt{x^{2}+\frac{m^{2}}{T^{2}}}\right)+1} .
$$

\section{APPENDIX B: THREE-POINT QED EXAMPLES}

We calculate the three-point function for QED. The current requires the computation of 12 graphs. We will write the current in terms of the following bases of tensor structures

$$
\overline{\mathcal{A}}^{\mu_{1} \mu_{2} \mu_{3}}(k, \bar{p})=\frac{2 e^{3}}{\left(k \cdot \bar{p}_{1}\right)^{2}} \sum_{i=1}^{23} \mathcal{T}_{i} a_{i}
$$

where

$$
\begin{aligned}
& a_{1}=\frac{\bar{p}_{2}{ }^{2} \bar{p}_{1} \cdot \bar{p}_{3}}{\left(k \cdot \bar{p}_{2}\right)^{2}}+\frac{\bar{p}_{3}{ }^{2} \bar{p}_{1} \cdot \bar{p}_{2}}{\left(k \cdot \bar{p}_{3}\right)^{2}}-\frac{2 \bar{p}_{1} \cdot \bar{p}_{2} \bar{p}_{1} \cdot \bar{p}_{3}}{k \cdot \bar{p}_{2} k \cdot \bar{p}_{3}}, \quad a_{2}=-\bar{p}_{1}{ }^{2}, \quad a_{3}=-\frac{\bar{p}_{2}{ }^{2}\left(k \cdot \bar{p}_{1}\right)^{2}}{\left(k \cdot \bar{p}_{2}\right)^{2}}, \\
& a_{4}=a_{12}=-k \cdot \bar{p}_{1}, \quad a_{5}=\frac{\left(k \cdot \bar{p}_{1}\right)^{2}}{k \cdot \bar{p}_{2}}, \quad a_{6}=a_{7}=-\frac{\bar{p}_{3}{ }^{2} k \cdot \bar{p}_{1}}{\left(k \cdot \bar{p}_{3}\right)^{2}}+\frac{\bar{p}_{1} \cdot \bar{p}_{3} k \cdot \bar{p}_{1}}{k \cdot \bar{p}_{2} k \cdot \bar{p}_{3}} \\
& a_{8}=\frac{\bar{p}_{2}{ }^{2} k \cdot \bar{p}_{3}}{\left(k \cdot \bar{p}_{2}\right)^{2}}+\frac{\bar{p}_{3}{ }^{2}}{k \cdot \bar{p}_{2}}-\frac{2 \bar{p}_{1} \cdot \bar{p}_{2}}{k \cdot \bar{p}_{2}}, \quad a_{9}=a_{10}=\frac{k \cdot \bar{p}_{1}}{k \cdot \bar{p}_{2}}, \quad a_{11}=-\frac{\bar{p}_{3}{ }^{2}\left(k \cdot \bar{p}_{1}\right)^{2}}{\left(k \cdot \bar{p}_{3}\right)^{2}}, \\
& a_{13}=a_{20}=-\frac{\bar{p}_{2}{ }^{2} k \cdot \bar{p}_{1}}{\left(k \cdot \bar{p}_{2}\right)^{2}}+\frac{\bar{p}_{1} \cdot \bar{p}_{2} k \cdot \bar{p}_{1}}{k \cdot \bar{p}_{2} k \cdot \bar{p}_{3}}, \quad a_{14}=a_{21}=a_{22}=-\frac{\left(k \cdot \bar{p}_{1}\right)^{2}}{k \cdot \bar{p}_{2} k \cdot \bar{p}_{3}}, \quad a_{15}=\frac{k \cdot \bar{p}_{1}}{k \cdot \bar{p}_{2}}, \\
& a_{16}=\frac{\bar{p}_{2}{ }^{2}}{k \cdot \bar{p}_{3}}+\frac{\bar{p}_{3}{ }^{2} k \cdot \bar{p}_{2}}{\left(k \cdot \bar{p}_{3}\right)^{2}}-\frac{2 \bar{p}_{1} \cdot \bar{p}_{3}}{k \cdot \bar{p}_{3}}, \quad a_{17}=a_{18}=a_{23}=\frac{k \cdot \bar{p}_{1}}{k \cdot \bar{p}_{3}}, \quad a_{19}=\frac{\left(k \cdot \bar{p}_{1}\right)^{2}}{k \cdot \bar{p}_{3}}
\end{aligned}
$$

and

$$
\begin{aligned}
& \mathcal{T}_{1}=k^{\mu_{1}} k^{\mu_{2}} k^{\mu_{3}}, \quad \mathcal{T}_{2}=\eta^{\mu_{2} \mu_{3}} k^{\mu_{1}}, \quad \mathcal{T}_{3}=\eta^{\mu_{1} \mu_{3}} k^{\mu_{2}}, \quad \mathcal{T}_{4}=\eta_{\mu_{2} \mu_{2}} \bar{p}_{2}{ }^{\mu_{1}}, \quad \mathcal{T}_{5}=\eta^{\mu_{1} \mu_{3}} \bar{p}_{2}{ }^{\mu_{2}}, \\
& \mathcal{T}_{6}=k^{\mu_{2}} k^{\mu_{3}} \bar{p}_{2}{ }^{\mu_{1}}, \quad \mathcal{T}_{7}=k^{\mu_{1}} k^{\mu_{3}} \bar{p}_{2}{ }^{\mu_{2}}, \quad \mathcal{T}_{8}=k^{\mu_{1}} k^{\mu_{2}} \bar{p}_{2}{ }^{\mu_{3}}, \quad \mathcal{T}_{9}=k^{\mu_{2}} \bar{p}_{2}{ }^{\mu_{1}} \bar{p}_{2}{ }^{\mu_{3}}, \quad \mathcal{T}_{10}=k^{\mu_{1}} \bar{p}_{2}{ }^{\mu_{2}} \bar{p}_{2}{ }^{\mu_{3}}, \\
& \mathcal{T}_{11}=\eta^{\mu_{1} \mu_{2}} k^{\mu_{3}}, \quad \mathcal{T}_{12}=\eta^{\mu_{2} \mu_{3}} \bar{p}_{3}{ }^{\mu_{1}}, \quad \mathcal{T}_{13}=k^{\mu_{2}} k^{\mu_{3}} \bar{p}_{3}{ }^{\mu_{1}}, \quad \mathcal{T}_{14}=k^{\mu_{3}} \bar{p}_{2}{ }^{\mu_{2}} \bar{p}_{3}{ }^{\mu_{1}}, \quad \mathcal{T}_{15}=k^{\mu_{2}} \bar{p}_{2}{ }^{\mu_{3}} \bar{p}_{3}{ }^{\mu_{1}}, \\
& \mathcal{T}_{16}=k^{\mu_{1}} k^{\mu_{3}} \bar{p}_{3}{ }^{\mu_{2}}, \quad \mathcal{T}_{17}=k^{\mu_{3}} \bar{p}_{2}{ }^{\mu_{1}} \bar{p}_{3}{ }^{\mu_{2}}, \quad \mathcal{T}_{18}=k^{\mu_{3}} \bar{p}_{3}{ }^{\mu_{1}} \bar{p}_{3}{ }^{\mu_{2}}, \quad \mathcal{T}_{19}=\eta^{\mu_{1} \mu_{2}} \bar{p}_{3}{ }^{\mu_{3}}, \\
& \mathcal{T}_{20}=k^{\mu_{1}} k^{\mu_{2}} \bar{p}_{3}{ }^{\mu_{3}}, \\
& \mathcal{T}_{21}=k^{\mu_{2}} \bar{p}_{2}{ }^{\mu_{1}} \bar{p}_{3}{ }^{\mu_{3}}, \quad \mathcal{T}_{22}=k^{\mu_{1}} \bar{p}_{2}{ }^{\mu_{2}} \bar{p}_{3}{ }^{\mu_{3}}, \quad \mathcal{T}_{23}=k^{\mu_{1}} \bar{p}_{3}{ }^{\mu_{2}} \bar{p}_{3}{ }^{\mu_{3}} .
\end{aligned}
$$

We have checked that this result is equivalent to the high temperature limit computed in Ref. [95]. The equivalence between these functions and solutions of the Abelian version of Eq. (5) is discussed Ref. [95]. 
[1] See Refs. [2-6] for reviews on the modern approach to scattering amplitudes and Ref. [7] for a review of the state of the art of multi-loop calculations.

[2] R. Britto, J. Phys. A 44, 454006 (2011).

[3] R. Ellis, Z. Kunszt, K. Melnikov, and G. Zanderighi, Phys. Rep. 518, 141 (2012).

[4] H. Elvang and Y.-t. Huang, arXiv:1308.1697.

[5] S. Weinzierl, Phys. Rep. 676, 1 (2017).

[6] C. Cheung, TASI lectures on scattering amplitudes, in Proceedings, Theoretical Advanced Study Institute in Elementary Particle Physics: Anticipating the Next Discoveries in Particle Physics (TASI 2016): Boulder, CO, USA, 2016, edited by R. Essig and I. Low (2018), pp. 571-623 [arXiv:1708.03872].

[7] G. Heinrich, Phys. Rep. 922, 1 (2021).

[8] S. Foffa, P. Mastrolia, R. Sturani, and C. Sturm, Phys. Rev. D 95, 104009 (2017).

[9] F. Cachazo and A. Guevara, J. High Energy Phys. 02 (2020) 181.

[10] C. Cheung, I. Z. Rothstein, and M. P. Solon, Phys. Rev. Lett. 121, 251101 (2018).

[11] D. A. Kosower, B. Maybee, and D. O'Connell, J. High Energy Phys. 02 (2019) 137.

[12] N. J. Bjerrum-Bohr, P. H. Damgaard, G. Festuccia, L. Planté, and P. Vanhove, Phys. Rev. Lett. 121, 171601 (2018).

[13] A. Guevara, A. Ochirov, and J. Vines, J. High Energy Phys. 09 (2019) 056.

[14] J. Blümlein, A. Maier, and P. Marquard, Phys. Lett. B 800, 135100 (2020).

[15] A. Cristofoli, N. Bjerrum-Bohr, P. H. Damgaard, and P. Vanhove, Phys. Rev. D 100, 084040 (2019).

[16] B. Maybee, D. O'Connell, and J. Vines, J. High Energy Phys. 12 (2019) 156.

[17] N. Arkani-Hamed, Y.-t. Huang, and D. O'Connell, J. High Energy Phys. 01 (2020) 046.

[18] Z. Bern, C. Cheung, R. Roiban, C.-H. Shen, M. P. Solon, and M. Zeng, Phys. Rev. Lett. 122, 201603 (2019).

[19] Z. Bern, C. Cheung, R. Roiban, C.-H. Shen, M. P. Solon, and M. Zeng, J. High Energy Phys. 10 (2019) 206.

[20] P. H. Damgaard, K. Haddad, and A. Helset, J. High Energy Phys. 11 (2019) 070.

[21] A. Brandhuber and G. Travaglini, J. High Energy Phys. 01 (2020) 010.

[22] Z. Bern, A. Luna, R. Roiban, C.-H. Shen, and M. Zeng, arXiv:2005.03071 [Phys. Rev. D (to be published)].

[23] R. Aoude, K. Haddad, and A. Helset, J. High Energy Phys. 05 (2020) 051.

[24] G. Mogull, J. Plefka, and J. Steinhoff, J. High Energy Phys. 02 (2021) 048.

[25] R. P. Feynman, Acta Phys. Pol. 24, 697 (1963).

[26] S. Catani, T. Gleisberg, F. Krauss, G. Rodrigo, and J.-C. Winter, J. High Energy Phys. 09 (2008) 065.

[27] S. Caron-Huot, J. High Energy Phys. 05 (2011) 080.

[28] I. Bierenbaum, S. Catani, P. Draggiotis, and G. Rodrigo, J. High Energy Phys. 10 (2010) 073.

[29] I. Bierenbaum, S. Buchta, P. Draggiotis, I. Malamos, and G. Rodrigo, J. High Energy Phys. 03 (2013) 025.

[30] S. Buchta, G. Chachamis, P. Draggiotis, I. Malamos, and G. Rodrigo, J. High Energy Phys. 11 (2014) 014.
[31] R. J. Hernandez-Pinto, G. F. R. Sborlini, and G. Rodrigo, J. High Energy Phys. 02 (2016) 044.

[32] R. Runkel, Z. Szőr, J. P. Vesga, and S. Weinzierl, Phys. Rev. Lett. 122, 111603 (2019); 123, 059902(E) (2019).

[33] R. Runkel, Z. Szőr, J. P. Vesga, and S. Weinzierl, Phys. Rev. D 101, 116014 (2020).

[34] J. J. Aguilera-Verdugo, R. J. Hernandez-Pinto, G. Rodrigo, G. F. Sborlini, and W. J. Torres Bobadilla, J. High Energy Phys. 01 (2021) 069.

[35] J. J. Aguilera-Verdugo, F. Driencourt-Mangin, R. J. Hernández-Pinto, J. Plenter, S. Ramirez-Uribe, A. E. Renteria Olivo, G. Rodrigo, G. F. Sborlini, W. J. Torres Bobadilla, and S. Tracz, Phys. Rev. Lett. 124, 211602 (2020).

[36] J. J. Aguilera-Verdugo, R. J. Hernandez-Pinto, G. Rodrigo, G. F. Sborlini, and W. J. Torres Bobadilla, J. High Energy Phys. 02 (2021) 112.

[37] A non-exhaustive sample references on the relation between loops and trees is Refs. [38-44].

[38] A. Brandhuber, B. Spence, and G. Travaglini, J. High Energy Phys. 01 (2006) 142.

[39] R. Huang, Q. Jin, J. Rao, K. Zhou, and B. Feng, J. High Energy Phys. 03 (2016) 057.

[40] B. Feng, S. He, R. Huang, and M.-x. Luo, J. High Energy Phys. 01 (2017) 008.

[41] F. Cachazo, S. He, and E. Y. Yuan, J. High Energy Phys. 08 (2016) 008.

[42] Y. Geyer, L. Mason, R. Monteiro, and P. Tourkine, Phys. Rev. Lett. 115, 121603 (2015).

[43] S. He and O. Schlotterer, Phys. Rev. Lett. 118, 161601 (2017).

[44] A. Ochirov, P. Tourkine, and P. Vanhove, J. High Energy Phys. 10 (2017) 105.

[45] F. T. Brandt, A. K. Das, J. Frenkel, and S. Perez, Phys. Rev. D 74, 125005 (2006).

[46] E. Braaten and R. D. Pisarski, Nucl. Phys. B337, 569 (1990).

[47] J. Frenkel and J. Taylor, Nucl. Phys. B334, 199 (1990).

[48] E. Braaten and R. D. Pisarski, Nucl. Phys. B339, 310 (1990).

[49] J. Taylor and S. Wong, Nucl. Phys. B346, 115 (1990).

[50] J. Frenkel and J. Taylor, Nucl. Phys. B374, 156 (1992).

[51] J. P. Blaizot and E. Iancu, Nucl. Phys. B417, 608 (1994).

[52] J.-P. Blaizot and E. Iancu, Nucl. Phys. B434, 662 (1995).

[53] P. Kelly, Q. Liu, C. Lucchesi, and C. Manuel, Phys. Rev. Lett. 72, 3461 (1994).

[54] A. Luna, I. Nicholson, D. O'Connell, and C. D. White, J. High Energy Phys. 03 (2018) 044.

[55] This approach has been reviewed in Refs. [56,57], see also Ref. [58] for a recent review.

[56] D. F. Litim and C. Manuel, Phys. Rep. 364, 451 (2002).

[57] J.-P. Blaizot and E. Iancu, Phys. Rep. 359, 355 (2002).

[58] S. Mrowczynski, B. Schenke, and M. Strickland, Phys. Rep. 682, 1 (2017).

[59] S. Wong, Nuovo Cimento A 65, 689 (1970).

[60] Notice that the measure is defined in terms of the dimensionless constant $c^{a} / \hbar$.

[61] L. S. Brown and W. I. Weisberger, Nucl. Phys. B157, 285 (1979); B172, 544(E) (1980).

[62] U. W. Heinz, Phys. Rev. Lett. 51, 351 (1983). 
[63] U. W. Heinz, Ann. Phys. (N.Y.) 161, 48 (1985).

[64] H.-T. Elze and U. W. Heinz, Phys. Rep. 183, 81 (1989).

[65] The term of order $\mathcal{O}\left(g^{0}\right)$ vanishes due to the identity $\int \mathrm{d} c c^{a}=0$.

[66] The current has to be understood as a functional of the soft gauge fields [51].

[67] M. Laine and A. Vuorinen, Basics of Thermal Field Theory, Vol. 925 (Springer, New York, 2016); Lect. Notes Phys. 925, 1 (2016).

[68] P. Kelly, Q. Liu, C. Lucchesi, and C. Manuel, Phys. Rev. D 50, 4209 (1994).

[69] W. D. Goldberger and A. K. Ridgway, Phys. Rev. D 95, 125010 (2017).

[70] T. Damour, Phys. Rev. D 97, 044038 (2018).

[71] W. D. Goldberger, S. G. Prabhu, and J. O. Thompson, Phys. Rev. D 96, 065009 (2017).

[72] W. D. Goldberger and A. K. Ridgway, Phys. Rev. D 97, 085019 (2018).

[73] W. D. Goldberger, J. Li, and S. G. Prabhu, Phys. Rev. D 97, 105018 (2018).

[74] D. Chester, Phys. Rev. D 97, 084025 (2018).

[75] C.-H. Shen, J. High Energy Phys. 11 (2018) 162.

[76] L. de la Cruz, B. Maybee, D. O'Connell, and A. Ross, J. High Energy Phys. 12 (2020) 076.

[77] See Ref. [76] for a longer discussion about this point.

[78] See e.g., Chap. 38 in Ref. [79].

[79] J. Zinn-Justin, Quantum Field Theory and Critical Phenomena, International Series of Monographs on Physics Vol. 77 (Oxford University Press, 1989).

[80] G. Barton, Ann. Phys. (N.Y.) 200, 271 (1990).

[81] See Ref. [82] for a simple derivation of the functional and Refs. [83-87] for the relation of the generating functional and Chern-Simons theory.

[82] P. Elmfors, T. Hansson, and I. Zahed, Phys. Rev. D 59, 045018 (1999).

[83] R. Efraty and V. Nair, Phys. Rev. Lett. 68, 2891 (1992).

[84] R. Efraty and V. Nair, Phys. Rev. D 47, 5601 (1993).

[85] R. Jackiw and V. Nair, Phys. Rev. D 48, 4991 (1993).

[86] V. Nair, Phys. Rev. D 48, R3432 (1993).

[87] V. Nair, Phys. Rev. D 50, 4201 (1994).

[88] F. T. Brandt and J. Frenkel, Phys. Rev. D 47, 4688 (1993).

[89] F. T. Brandt and J. Frenkel, Phys. Rev. D 48, 4940 (1993).

[90] F. T. Brandt and J. Frenkel, Phys. Rev. D 56, 2453 (1997).

[91] F. T. Brandt, A. K. Das, J. Frenkel, and A. da Silva, Phys. Rev. D 59, 065004 (1999).

[92] F. T. Brandt, A. K. Das, and J. Frenkel, Phys. Rev. D 60, 105008 (1999).

[93] F. Brandt, J. Frenkel, and D. McKeon, Phys. Rev. D 65, 125029 (2002).

[94] F. Brandt, A. K. Das, and J. Frenkel, Phys. Rev. D 66, 105012 (2002).

[95] F. Brandt, R. Ferreira, and J. Thuorst, Phys. Rev. D 91, 045023 (2015).

[96] See Ref. [33] for a general version of this idea within looptree duality.

[97] F. A. Berends and W. Giele, Nucl. Phys. B306, 759 (1988).
[98] A. K. Das, Finite Temperature Field Theory (World Scientific, New York, 1997).

[99] We thank Fernando T. Brandt for this insight.

[100] See Ref. [101] for the use of scalar QED as a toy model for gluonic QCD.

[101] U. Kraemmer, A. Rebhan, and H. Schulz, Ann. Phys. (N.Y.) 238, 286 (1995).

[102] M. L. Bellac, Thermal Field Theory, Cambridge Monographs on Mathematical Physics (Cambridge University Press, Cambridge, England, 2011).

[103] The contribution from three-gluon vertex is typically neglected because it does not correspond to a one-particle irreducible graph.

[104] F. Brandt, A. K. Das, J. Frenkel, D. McKeon, and J. Taylor, Phys. Rev. D 66, 045011 (2002).

[105] U. Kraemmer and A. Rebhan, Rep. Prog. Phys. 67, 351 (2004).

[106] F. T. Brandt and J. Frenkel, Phys. Rev. D 58, 085012 (1998).

[107] B. R. Holstein, Am. J. Phys. 74, 1002 (2006).

[108] R. Britto, F. Cachazo, B. Feng, and E. Witten, Phys. Rev. Lett. 94, 181602 (2005).

[109] Z. Bern, J. J. M. Carrasco, and H. Johansson, Phys. Rev. D 78, 085011 (2008).

[110] Z. Bern, J. J. M. Carrasco, and H. Johansson, Phys. Rev. Lett. 105, 061602 (2010).

[111] Z. Bern, J. J. Carrasco, M. Chiodaroli, H. Johansson, and R. Roiban, arXiv:1909.01358.

[112] R. Britto and A. Ochirov, J. High Energy Phys. 01 (2013) 002.

[113] P. Mastrolia, A. Primo, U. Schubert, and W. J. Torres Bobadilla, Phys. Lett. B 753, 242 (2016).

[114] J. L. Jurado, G. Rodrigo, and W. J. Torres Bobadilla, J. High Energy Phys. 12 (2017) 122.

[115] R. Monteiro, D. O'Connell, and C. D. White, J. High Energy Phys. 12 (2014) 056.

[116] Collision functions in the context of classical transport theory have been studied e.g., in Refs. [117-119].

[117] D. F. Litim and C. Manuel, Phys. Rev. Lett. 82, 4981 (1999).

[118] D. F. Litim and C. Manuel, Nucl. Phys. B562, 237 (1999).

[119] D. F. Litim and C. Manuel, Phys. Rev. D 61, 125004 (2000).

[120] D.-L. Yang, K. Hattori, and Y. Hidaka, J. High Energy Phys. 07 (2020) 070.

[121] N. Weickgenannt, E. Speranza, X.-1. Sheng, Q. Wang, and D. H. Rischke, arXiv:2005.01506.

[122] S. De Groot, in Relativistic Kinetic Theory. Principles and Applications, edited by W. Van Leeuwen and C. Van Weert (1980).

[123] J. Ellis, Comput. Phys. Commun. 210, 103 (2017).

[124] R. Mertig, M. Bohm, and A. Denner, Comput. Phys. Commun. 64, 345 (1991).

[125] V. Shtabovenko, R. Mertig, and F. Orellana, Comput. Phys. Commun. 207, 432 (2016).

[126] V. Shtabovenko, R. Mertig, and F. Orellana, Comput. Phys. Commun. 256, 107478 (2020). 